\title{
Integrated metagenomic data analysis demonstrates that a loss of diversity in oral microbiota is associated with periodontitis
}

\author{
Dongmei $\mathrm{Ai}^{1 \dagger}$, Ruocheng Huang ${ }^{1 \dagger}$, Jin Wen ${ }^{2,3}$, Chao Li ${ }^{1}$, Jiangping Zhu ${ }^{1}$ and Li Charlie Xia ${ }^{4,5^{*}}$
}

From The 27th International Conference on Genome Informatics

Shanghai, China. 3-5 October 2016

\begin{abstract}
Background: Periodontitis is an inflammatory disease affecting the tissues supporting teeth (periodontium). Integrative analysis of metagenomic samples from multiple periodontitis studies is a powerful way to examine microbiota diversity and interactions within host oral cavity.

Methods: A total of 43 subjects were recruited to participate in two previous studies profiling the microbial community of human subgingival plaque samples using shotgun metagenomic sequencing. We integrated metagenomic sequence data from those two studies, including six healthy controls, 14 sites representative of stable periodontitis, 16 sites representative of progressing periodontitis, and seven periodontal sites of unknown status. We applied phylogenetic diversity, differential abundance, and network analyses, as well as clustering, to the integrated dataset to compare microbiological community profiles among the different disease states.

Results: We found alpha-diversity, i.e., mean species diversity in sites or habitats at a local scale, to be the single strongest predictor of subjects' periodontitis status $(P<0.011)$. More specifically, healthy subjects had the highest alpha-diversity, while subjects with stable sites had the lowest alpha-diversity. From these results, we developed an alpha-diversity logistic model-based naive classifier able to perfectly predict the disease status of the seven subjects with unknown periodontal status (not used in training). Phylogenetic profiling resulted in the discovery of nine marker microbes, and these species are able to differentiate between stable and progressing periodontitis, achieving an accuracy of $94.4 \%$. Finally, we found that the reduction of negatively correlated species is a notable signature of disease progression.

Conclusions: Our results consistently show a strong association between the loss of oral microbiota diversity and the progression of periodontitis, suggesting that metagenomics sequencing and phylogenetic profiling are predictive of early periodontitis, leading to potential therapeutic intervention. Our results also support a keystone pathogen-mediated polymicrobial synergy and dysbiosis (PSD) model to explain the etiology of periodontitis. Apart from $P$. gingivalis, we identified three additional keystone species potentially mediating the progression of periodontitis progression based on pathogenic characteristics similar to those of known keystone pathogens.
\end{abstract}

\footnotetext{
* Correspondence: lixia@stanford.edu

${ }^{\dagger}$ Equal contributors

${ }^{4}$ Department of Medicine, Stanford University School of Medicine, 269

Campus Dr., Stanford, CA 94305, USA

${ }^{5}$ Department of Statistics, The Wharton School, University of Pennsylvania,

3730 Walnut Street, Philadelphia, PA 19014, USA

Full list of author information is available at the end of the article
} 


\section{Background}

Periodontitis results from the hyperimmune response of our body toward pathogenic bacteria resident in the oral cavity, which causes the destruction of periodontal connective tissue [1]. Periodontitis can increase the risk of such systemic conditions as cardiovascular disease, diabetes and obesity [2-4]. According to the latest epidemiological data, more than $47 \%$ of U.S. adults suffer from periodontal diseases, including gingivitis and periodontitis [5]. It is generally accepted that the presence of pathogenic bacterial species in host oral cavity, contributes to the onset and development of periodontal diseases. In fact, more than 700 oral microbial phylotypes have already been identified by cultivation, traditional cloning and sequencing $[6,7]$. Nonetheless, the exact etiology of periodontal disease, in particular, periodontitis, is yet to be determined.

In earlier years, the etiology of periodontitis was attributed to a few specific plaque species of oral microbiota [8]. For example, using in vitro culture and checkerboard DNA-DNA hybridization, the "red complex" was identified. It consisted of Porphyromonas gingivalis, Treponema denticola and Tannerella forsythia, which are considered to be the most virulent organisms involved in the etiology of periodontitis $[9,10]$. Later, Kumar et al. [11] used species-specific 16S rRNA sequencing to expand the catalogue of periodontal pathogens, and the results suggested that periodontitis arises from nonspecific inflammation with diverse progression patterns in response to various plaque species [12]. Then, Marsh et al. proposed that periodontitis is caused by an imbalance of microflora resulting from ecological stress, in turn, enriching the presence of disease-related microorganisms $[13,14]$.

However, culture-based methods have practical limitations and may overestimate the abundance microbes, resulting in biased estimates. Similarly, species-specific techniques capture only a small fraction of the extremely diverse and complex human oral microbiome. Moreover, neither method can systematically characterize how dental plaque (biofilm) causes destruction of the toothsupporting structures in the inflammatory state. Recently, the advancement of "omics" technologies has enabled a more holistic approach to the assessment of host oral microbiota. Specifically, it is only with the advent of culture-free, high-throughput sequencing technologies, such as 16S rRNA and shotgun metagenomic sequencing, that we can now comprehensively characterize and compare constituents of bacterial communities with unprecedented resolution. Recent widespread adoption of next-generation sequencing (NGS) technologies has led to even more massive, albeit short, metagenomic datasets $[15,16]$.

NGS metagenomic sequencing has produced a rich abundance of information about microbial communities compared to traditional sequencing data because of the significant increase in read depth. Previous studies using NGS metagenomic analysis have already advanced our understanding of periodontitis. Based on $16 \mathrm{~S}$ rRNA and shotgun sequencing, studies like Loreto et al. [17] and Wang et al.[18] have confirmed significant differences in microbial community structures between healthy and periodontally compromised subjects. Orth et al. later used a combination of culture-based methods and highthroughput sequencing to identify a keystone pathogen, Porphyromonas gingivalis, which, although prevalent in subgingival samples, can influence host immune response to promote the bacteria that cause periodontitis [19].

As noted above, no consensus has thus far been reached to explain the exact etiology of periodontitis. Therefore, to gain further insight into the composition and structure of oral microbial communities in the context of disease onset, this study first integrated metagenomic sequence data from two previous studies that profiled the microbial community of human subgingival plaque samples, including in total six healthy controls and 37 periodontally diseased samples (among which 14 represent stable periodontitis, 16 represent progressing periodontitis, and the remaining seven samples are diseased but without further classified as stable or progressing. Next, phylogenetic diversity, differential abundance, and network analyses, as well as clustering, were applied to this integrated dataset to compare microbiological community profiles among the different disease states. Accordingly, the paper is organized into three main sections to (1) describe the procedures and software pipeline used for analysis, (2) identify and compare differentially represented microbial species between healthy control and periodontitis subjects, both stable and progressing, using alpha-diversity as the key metric, and (3) cluster species profiles to identify additional keystone species and compare the structure of oral microbial co-occurrence correlation networks using network analysis.

\section{Methods}

\section{Integration of periodontitis metagenomic datasets}

In this study, we first curated and integrated datasets published earlier by Duran-Pinedo et al. and Yost et al. $[20,21]$, respectively. These studies analyzed gene ontology and phylogenetic composition, as well as catalogued the relevant activities of bacteria in samples with and without periodontitis. However, they did not statistically analyze key factors such as ecological diversity, composition similarity and co-occurrence networks that would have otherwise allowed us to understand the relationship between diversity in the microbial community and the disease state. 
This type of study could only be accomplished through the use of a more powerful and integrated comparative metagenomic analysis combining samples from multiple datasets. Owing to high cost, metagenomics projects are typically based on a small number of samples, which limits the power of statistical analysis. Integrating raw data from multiple projects with standardized bioinformatics pipeline would allow us to increase the sample size and boost the statistical power. In this study, by combining data of 13 and 30 samples from two original studies, we arrived at a total of 43 samples, a much larger number and with both healthy and diseased samples. The integrated analysis also allows us to systemically identify the marker and keystone species and exam the co-occurrence networks. Such results were not present in the original studies.

More specifically, we collected all whole genome shotgun sequenced (Illumina sequencing) metagenomic samples from those two studies, which include six controls of metagenomic samples taken from subgingival plaques of healthy individuals, and 37 cases from periodontitis patients. Among the 37 periodontitis metagenomic datasets, 14 samples were from subjects in stable status, as determined by Clinical Attachment Loss (CAL) of $<2 \mathrm{~mm}$ compared to their last visit. Sixteen samples were in progressing status, having CAL $>2 \mathrm{~mm}$. Seven samples were from subjects with periodontitis, but their status was unknown. To clarify the terms we used, the disease "state" is either healthy or periodontitis, while the disease "status" can be stable, progressing or unknown.

\section{Bioinformatics pipeline for integrated metagenomics analysis}

We constructed a bioinformatics pipeline (Fig. 1) consisting of six steps, as follows: (1) Quality Control and

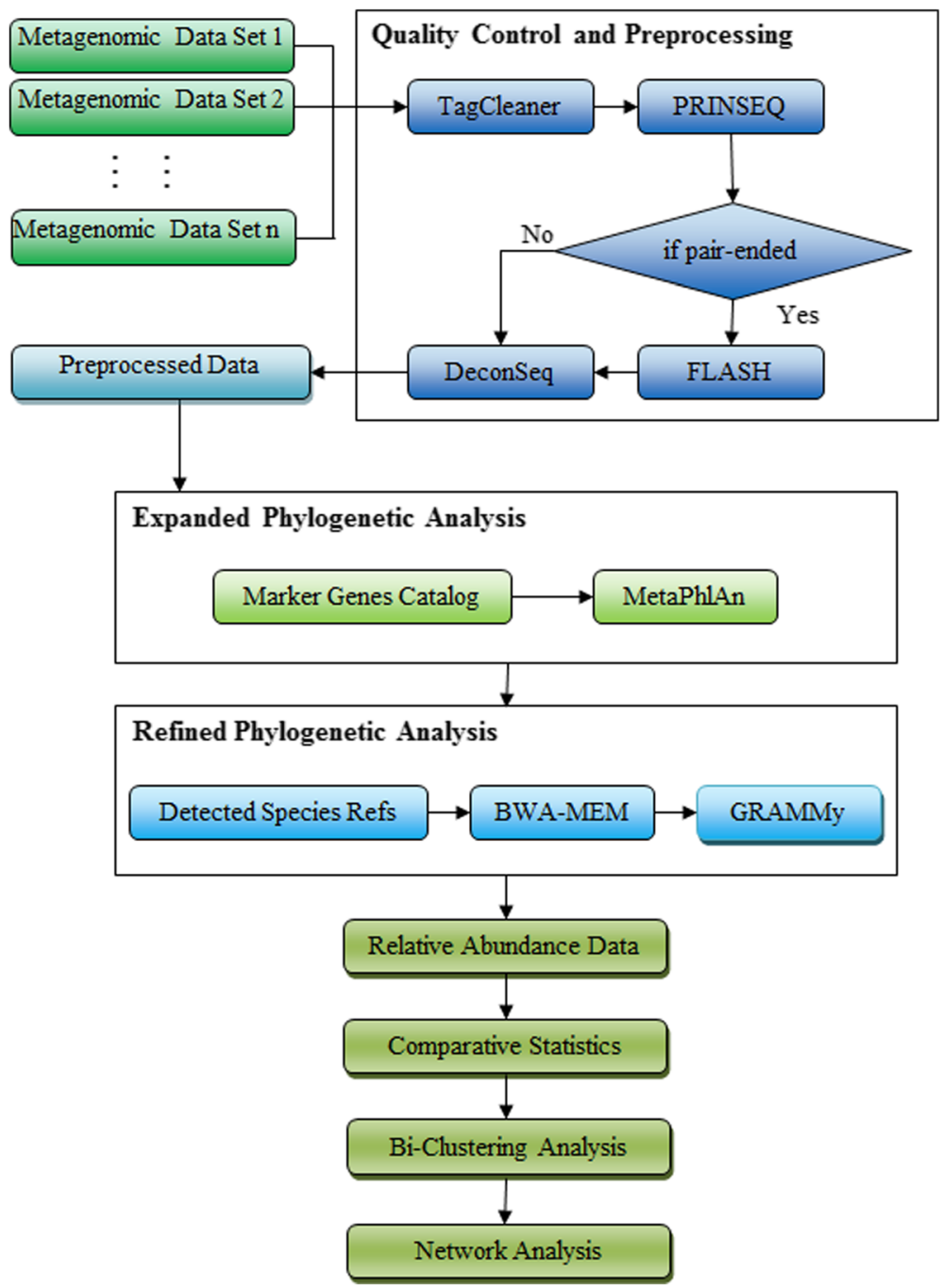

Fig. 1 Data preprocessing and bioinformatics pipeline for integrated metagenomics analysis 
Preprocessing, in which TagCleaner, PRINSEQ, Deconseq and FLASH [22-25] were used to remove low quality reads and contamination from the human genome;

(2) Expanded Phylogenetic Analysis, in which MetaPhlAn [26] was used to sensitively detect the presence of microbial species inoral samples; (3) Refined Phylogenetic Analysis, in which GRAMMy [27] was used to accurately estimate the relative abundance of the detected microbial species; (4) Statistical Analysis, in which the Dunn test was applied to compare the relative abundance of species and alpha-diversity of microbial communities based on different periodontitis states; (5) Clustering Analysis, in which individual oral samples were clustered based on the similarity of marker species abundance profiles; and (6) Network Analysis, in which co-occurrence correlation networks based on different periodontitis states were inferred and compared.

\section{Quality control and preprocessing of metagenomic reads} TagCleaner [22] was used to remove sequencing tags. Tags were predicted by TagCleaner with coverage over $50 \%$. Read sequences at either end representing tags without mismatches were removed. PRINSEQ [23] was then used to remove low-quality reads. Those reads with mean quality score lower than 15 , or with a read length out of the range of $3 \sigma$ from the mean read length, or with more than $1 \%$ missing base pairs (bp), were filtered out. Duplicate sequences were also removed. DeconSeq [24] was next used to remove contaminated reads originating from the human genome, i.e., those reads mapped to the human genome with over 98\% identity and over $98 \%$ base pairs aligned. Finally, FLASH [25] was employed to merge pair-ended reads where paired reads were removed if their overlaps were over $65 \mathrm{bp}$.

\section{Expanded phylogenetic analysis}

A total of 43 metagenomes sampled from healthy and periodontitis subgingival plaques were analyzed using MetaPhlAn [26], which mapped metagenomic reads to a marker gene catalogue and identified oral microbiota species inhabiting sample environments based on all available reference genomes from the Integrated Microbial Genomes (IMG) system [28]. Expanded phylogenetic analysis allows us to explore the tens of thousands reference species and narrow them down to specific species that are most relevant to our metagenomic samples.

\section{Refined phylogenetic analysis}

GRAMMy [27] was used to estimate the relative abundance of microbes present in the oral sample as identified in the expanded phylogenetic analysis. The complete genomes of present archaea and bacteria, as detected by MetaPhlAn, were downloaded from the Human Oral Microbiome Database [29] to construct the refined reference set for GRAMMy analysis. BWA-MEM [30] was used to align those metagenomic reads that passed the quality filtering to the reference sets. The alignment parameters were set to default, i.e., minimum seed length was set to 19 and mismatch penalty score was set to four, and all plausible alignments were output.

We then applied GRAMMy to the resulting BAM files to estimate oral microbial composition for subgingival plaque samples. GRAMMy was set to default parameters where the e-value threshold was $10 \mathrm{e}-5$, the alignment length threshold was $75 \mathrm{bp}$, and the identity threshold was $75 \%$. We then used the obtained abundance profiles for the downstream analysis, including, for example, alpha-diversity calculation, statistical testing, biclustering, and network analysis.

\section{Differential phylogenetic analysis}

To identify microbial species differentially present in healthy samples, as well as stable and progressing periodontitis, we applied the Dunn test to compare the relative abundances of detected microbial species (dunn.test in the stats package of $\mathrm{R})$. We adjusted the Dunn test $p$ values by Benjamini-Hochberg $(\mathrm{B}-\mathrm{H})$ correction to control false discovery (p.adjust in the stats package of R) [31].

\section{Alpha diversity analysis}

We used the Dunn test, as described above, to compare samples from healthy control, as well as stable and progressing periodontitis, relative to differences in microbial community alpha-diversity. We used Shannon index to measure the alpha-diversity of host oral community. Shannon index is defined as,

$$
H=\sum_{j=1}^{N} a_{j} \log a_{j}
$$

where $N$ represents the total number of detected species, and $a_{j}$ is the relative abundance of the $j$-th species.

In order to test for the potential association between oral microbial community diversity and periodontitis, we performed univariate logistic regression analysis by modeling microbial alpha-diversity as a factor contributing to the probability of developing periodontitis. The model was trained on the six healthy control and 30 periodontal samples whose status, e.g. stable or progressing, were already known. We then used the fitted logistic model as a naïve classifier to predict the potential of developing periodontitis among those remained seven periodontal metagenomic samples whose status was originally unknown and, hence, not part of the fitting data. To run the logistic regression analysis, we used the $g l m$ function in the stats package of R. 


\section{Biclustering analysis}

We used the heatmap. 2 function in the gplots package of $\mathrm{R}$ to bicluster and visually display microbial abundance profiles based on healthy and periodontitis mategenomic samples. In order to generate dendrograms for heatmaps, we applied a chi-square transfromation (decostan function of vegan package in R). The formula is as follows,

$$
\frac{a_{i j}^{\prime}=a_{i j}}{a_{i} \cdot \sqrt{a \cdot j}}
$$

where $a_{i}$ is the sum over columns (species), which should be one in relative abundance data matrix, and $a_{. j}$ is the sum over rows (samples). By applying chi-square transformation before ordinary biclustering, we can obtain more reasonable distances among metagenomic samples when the data are sparse [32].

We then calculated the Spearman correlations between samples based on differential relative abundances of represented species, using the cor function in the stats package of R. We converted the correlations to distances by

$$
\text { dist }=1-\text { cor }
$$

and generated the hierarchical clusters of the samples using the hclust function (method="average") in the same $\mathrm{R}$ package, which were then automatically converted to dendrograms in the heatmap.2 function [33]. The "average" method clusters samples by considering the average distance of any member of one cluster to any member of the other cluster.

\section{Co-occurrence correlation network analysis}

Co-occurrence correlation networks can reveal multipartner microbial interactions [34-38]. To characterize such networks in healthy control, as well as stable and progressing periodontitis samples, we calculated the global Spearman correlations of relative abundances for all pairs of microbial species detected under different states of periodontitis. The $p$-values were adjusted by Benjamini-Hochberg correction. Positive and negative links were drawn between pairs of species whose adjusted $p$-values were less than 0.05 . We used the igraph package of $\mathrm{R}$ to visualize networks under different states of periodontitis.

\section{Results}

Variability of the most abundant species in periodontitis samples

After preprocessing, healthy samples included an average number of 1,480,414 reads with an average length of 145 bp. Stable samples contained 1,502,809 reads with an average read length of $95 \mathrm{bp}$, whereas progressing periodontitis samples consisted of an average 746,776 reads and an average read length of $300 \mathrm{bp}$. The heterogeneity in read length can be attributed to different sequencing run configurations such as $2 * 150$ and 2 *250 cycles used in the original studies [20, 21]. This sequencing heterogeneity had no effect on our downstream analysis.

From the initial expanded phylogenetic analysis, 135 microbial species were identified by MetaPhlAn. A total of 396 genomes of those species were downloaded from HOMD and used as references for refined phylogenetic analyses. On average, we retrieved three complete genomes for each oral species in the reference set. We used BWA-MEM to map metagenomic reads to references and then used GRAMMy to estimate the relative abundances based on BWA mappings. From healthy and periodontitis metagenomic samples, a total of 70 microbial species were found to have detectable relative abundance by GRAMMy. On average, abundance levels of 47, 31 and 34 microbial species were detected by GRAMMy in subgingival samples from healthy, stable and progressing periodontitis sites, respectively.

Figure 2 shows the most abundant microbial species across healthy, stable and progressing subgingival samples. The top ten species in healthy control account for $75.8 \%$ (with $\mathrm{SD}=11.1 \%$ ) of total abundance in healthy samples, while total abundance for the top ten species is $87.1 \%$ (with $\mathrm{SD}=20.9 \%$ ) for progressing samples and $80.1 \%$ (with $\mathrm{SD}=18.9 \%$ ) for stable samples. The proportions of the top ten species in these three groups are significantly different $(P=6.61 \mathrm{e}-10$, the prop.test function from the stats package in $R$ ). That is the species not in top 10 account for significantly more proportion in healthy samples. In this figure, it can be seen that species from Streptococcus and Rothia are the most abundant microbes across all healthy, stable and progressing subgingival sites and that they are predominant in the human oral microbiome under both healthy and periodontitis conditions, as expected.

Among other abundant species, periodontitis samples, either stable or progressing, share another three genera, including Atopobium, Lactobacillus and Staphylococcus, while the samples from healthy control and progressing periodontitis oral sites share only one other abundant genus: Gemella. On the other hand, samples from healthy and stable periodontitis sites share only Streptococcus and Rothia. The remaining abundant species specific to healthy samples are from Actinomyces, Filifactor, Haemophilus, and Propionibacterium. Of the remaining abundant genera, those specific to progressing periodontitis samples are Bulleidia and Olsenella, while those specific to stable samples are Campylobacter and Eubacterium.

It is notable that the abundance distribution of the top ten species is more variable in stable (3 species with 


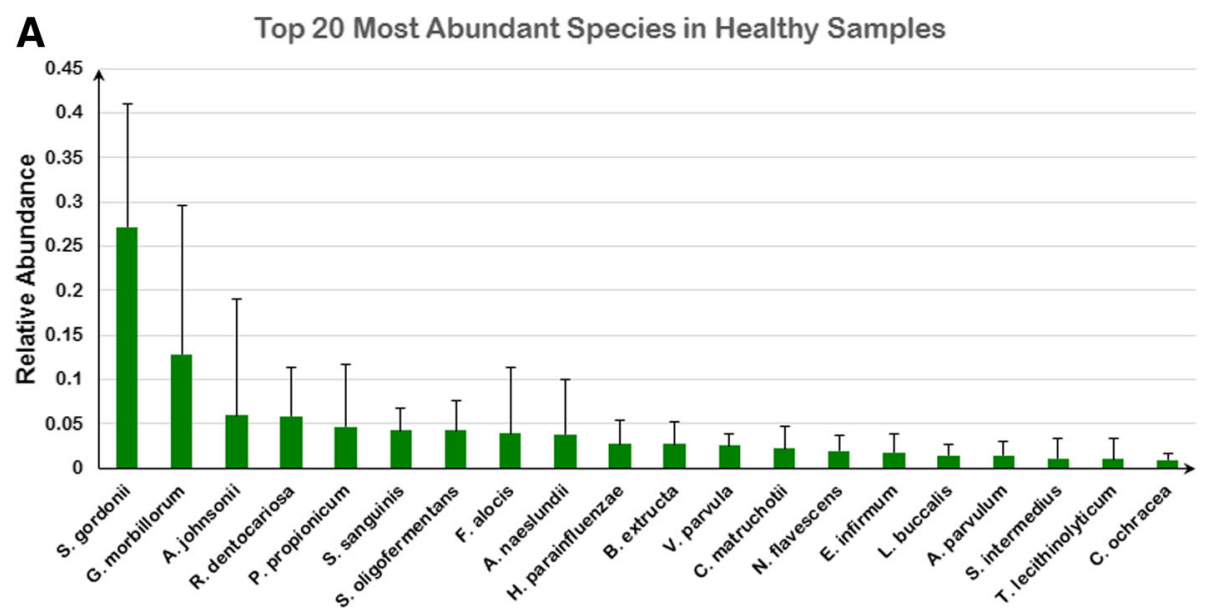

B Top 20 Most Abundant Species in Stable Periodontitis Samples

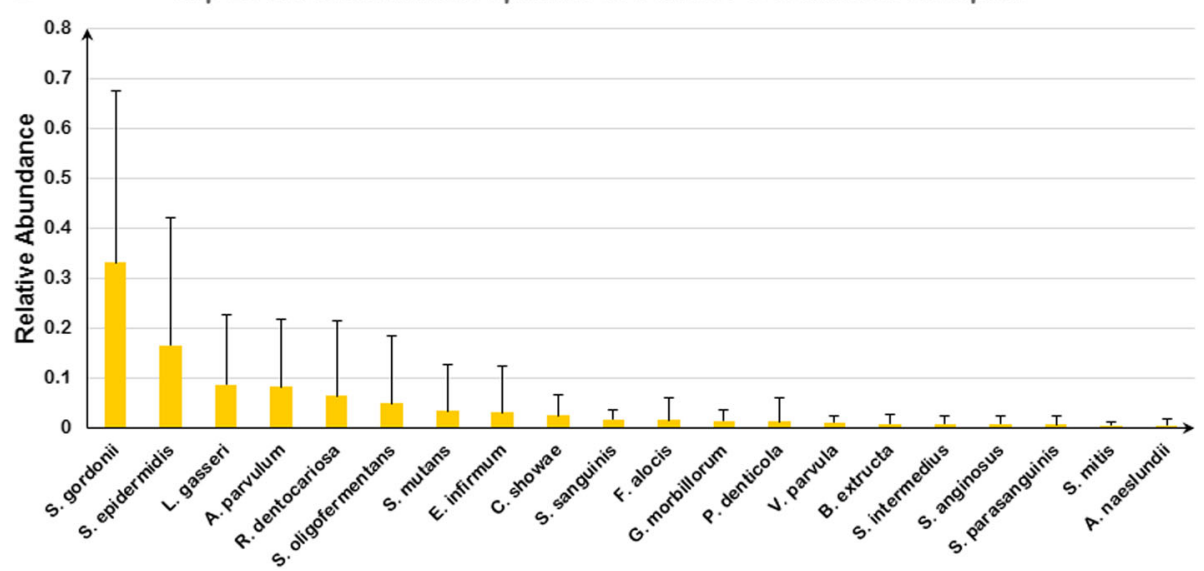

C Top 20 Most Abundant Species in Progressing Periodontitis Samples

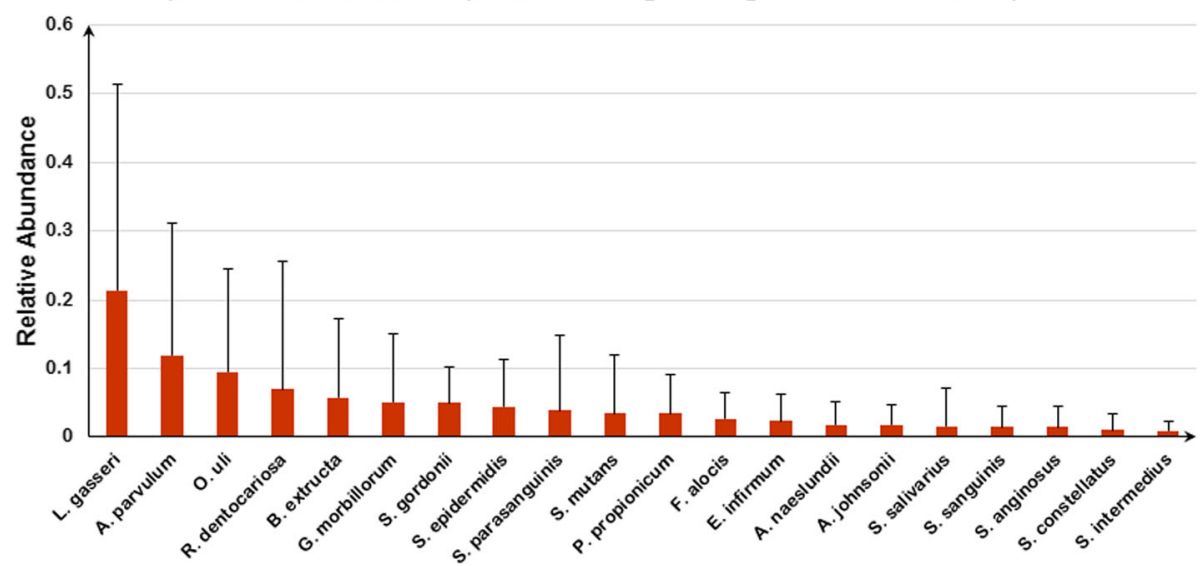

Fig. 2 Top 20 most abundant species of human subgingival plaque microbiota. The boxplots of top 20 most averagely abundant microbial species across samples taken from subgingival plaques under different periodontitis states. The same genus is shown in the same color. a represents those species in healthy samples, $\mathbf{b}$ ) represents those in stable samples and $\mathbf{c}$ ) represents progressing samples 
$\mathrm{SD}>+/-15 \%$ and average $\mathrm{SD}=14.5 \%)$ or progressing samples (4 species with SD $>+/-15 \%$ and average SD of $=13.6 \%)$, when compared to healthy control samples (only one species with $\mathrm{SD}>+/-15 \%$ and average $\mathrm{SD}=$ 7.9\%) (see Fig. 2). In addition, more outliers are found among the top 10 most abundant microbes of stable and progressing samples compared to healthy control samples. Importantly, these observations show a significant reduction of overall ecological diversity in the periodontitis samples, as demonstrated by the concentration of abundance toward only a few dominant species.

\section{Differentially abundant marker species in periodontitis samples}

We found nine marker species whose relative abundances were significantly different among healthy $(\mathrm{H})$, stable (S) and progressing (P) periodontitis sites, as shown in Fig. 3b. We found that Lactobacillus gasseri (Dunn test, $(\mathrm{H}$ vs. $\mathrm{P}), P=0.014)$, Campylobacter showae (Dunn test, (H vs. P), $P=0.034$ ) and Streptococcus sanguinis (Dunn test, $(\mathrm{H}$ vs. $\mathrm{P}$ ), $P=0.008$ ) were significantly different in progressing periodontitis samples compared to healthy samples. Among them, Lactobacillus gasseri was more abundant in progressing samples, while Campylobacter showae and Streptococcus sanguinis were more abundant in healthy samples.

Five more species had significantly higher relative abundance in healthy samples compared to periodontitis samples, both stable and progressing. Among them, Gemella morbillorum (Dunn test, ( $\mathrm{H}$ vs. $\mathrm{S}$ ), $\mathrm{P}=0.010$ and (H vs. $\mathrm{P}), P=0.009$ ) and Veillonella parvula (Dunn test, $(\mathrm{H}$ vs. $\mathrm{S}), \mathrm{P}=0.028$ and $(\mathrm{H}$ vs. $\mathrm{P}), P=0.007)$ were found in both healthy and periodontitis samples, while Haemophilus parainfluenzae (Dunn test, (H vs. S), $P<$ 0.001 and (H vs. $\mathrm{P}$ ), $P<0.001$ ), Corynebacterium matruchotii (Dunn test, (H vs. S), $P=0.016$ and (H vs. P), $P=$ 0.004 ) and Neisseria flavescens (Dunn test, (H vs. S), $P<$ 0.001 and $(\mathrm{H}$ vs. $\mathrm{P}), P<0.001)$ were only found in healthy samples. The statistical significance of Dunn tests is also shown in Fig. 3b.

The results suggest that they are marker species can be used in biclustering to differentiate among periodontitis states, as discussed later. In addition, Lactobacillus gasseri (Dunn test, ( $\mathrm{P}$ vs. S), $P=0.049$ ), Osenella uli (Dunn test, (P vs. S), $P=0.002$ ), and Campylobacter showae (Dunn test, (P vs. S), $P<0.001$ ) can differentiate between stable and progressing periodontitis, where the first two species were significantly higher in abundance in progressing periodontitis, and the last species was significantly lower.

\section{Microbial community alpha-diversity predicts disease status}

Alpha-diversity measures the biological diversity of a community, taking both species richness and variance in species proportion into consideration. Using Shannon index as the metric for alpha-diversity, we found the average to be 2.313 for healthy samples, 1.672 for progressing samples, and 1.329 for stable samples. The alpha-diversity of healthy samples is higher than that of progressing samples (Dunn test, $P=0.012$ ) and stable samples (Dunn test, $P<0.001$ ). However, alpha-diversity

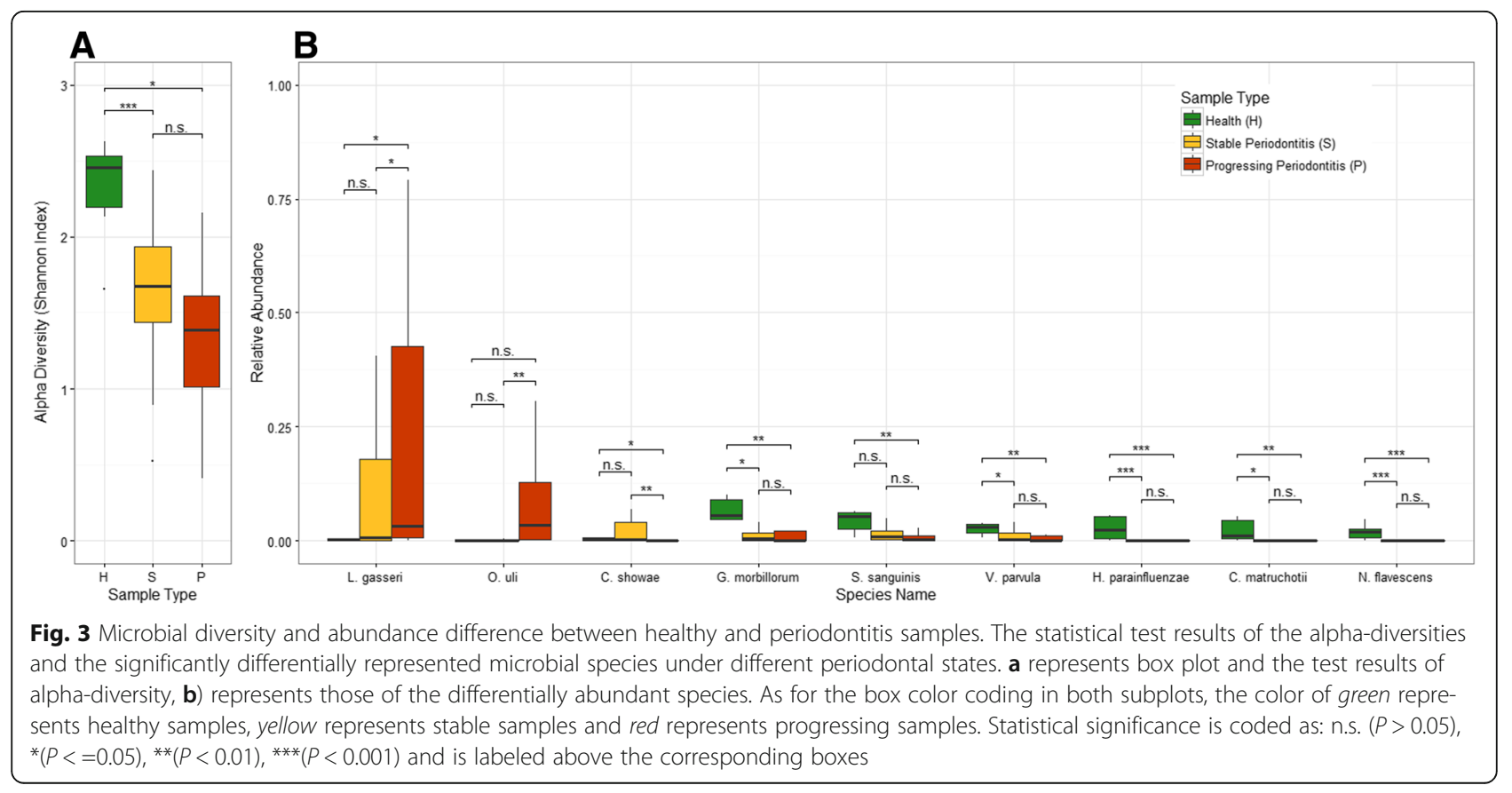


of progressing samples is not significantly higher than that of stable samples (Dunn test, $P=0.066$ ), which had the lowest alpha-diversity (Fig. 3a).

In order to see if alpha-diversity could be used as a predictor of periodontitis, we fitted a univariate logistic regression model with alpha-diversity as the independent variable and the probability of disease status as the response variable. The fitted values are in Table 1 , and the final model is

$$
\log \left(\frac{p}{1-p}\right)=-4.343 d+10.212
$$

where $p$ represents the probability of an individual having periodontitis, and $d$ represents the oral microbial alpha-diversity of the oral microbiome. It can be seen that the coefficient for alpha-diversity in this logistic model is negative, which means that the odds ratio is less than 1 . Therefore, the decrease in alpha-diversity of oral microbiome correlates with a higher probability of periodontitis.

The fitted model was then used as a naïve classifier to predict the periodontitis state of seven previously unclassified periodontal samples, which were not used in the fitting. The prediction results, which are found in Table 2, show that six out of the seven subjects were predicted as having periodontitis with high probabilities over 0.7. The remaining subject also had a greater than $50 \%$ chance of having periodontitis. If disease status were called as the most probable inference from the model, we would have $100 \%$ accuracy.

\section{Biclustering of community profiles and species in health and periodontitis samples}

The abundance profiles of 70 microbial species from all samples are shown as a heatmap in Fig. 4. Here, rows are clustered based on Spearman Rank-Order Correlations between the profiles of detected marker species, and columns are clustered for sample abundance similarity between microbial species. We see that all samples from healthy sites are perfectly clustered into one group and that all periodontitis samples are clustered into another group. Moreover, within the periodontitis group, most stable and progressing samples are clustered into subgroups. These results suggest that rank transformed abundance levels are strong predictors of healthy, stable and progressing periodontitis status.

Table 1 The fitted logistic regression model for periodontitis status and Alpha-diversity

\begin{tabular}{lccrc}
\hline & Estimate & Std. Error & zvalue & $\operatorname{Pr}(>|z|)$ \\
\hline Intercept & 10.212 & 3.732 & 2.736 & 0.00621 \\
Alpha-diversity & -4.343 & 1.694 & -2.564 & 0.01035 \\
\hline
\end{tabular}

Table 2 Predicted periodontitis probabilities for unknown state patients using the fitted logistic model

\begin{tabular}{lcc}
\hline Sample & Alpha-diversity & Predicted prob. \\
\hline Patient 1 & 2.326 & 0.528 \\
Patient 2 & 1.277 & 0.991 \\
Patient 3 & 1.606 & 0.962 \\
Patient 4 & 2.086 & 0.760 \\
Patient 5 & 1.917 & 0.869 \\
Patient 6 & 1.375 & 0.986 \\
Patient 7 & 1.794 & 0.919 \\
\hline
\end{tabular}

With column clustering, it should be noted that Porphyromonas gingivalis, previously known as a keystone pathogen [39], is clustered into a small group with Haemophilus haemolyticus, Prevotella melaninogenica and Capnocytophaga ochracea, indicating that these microbial species have an abundance profile similar to that of Porphyromonas gingivalis, thus further suggesting that these species may also play a role as keystone pathogens. The overall distribution by heatmapping intuitively shows these microbial species to be more diverse, i.e., more uniformly distributed, in healthy samples compared to those in stable or progressing samples.

\section{Patterns of community networks in healthy and periodontitis samples}

Finally, we inferred the co-occurrence correlation networks of oral microbial communities inhabiting subgingival plaques under different status of periodontitis based on the Spearman correlations of oral species pairs. In the network shown in Fig. 5, all the species pairs with FDR $<0.05$ were drawn. They all have a relatively high correlation (correlation absolute value $>0.8$ ). 21 positive (red-colored edges) and seven negative correlations (blue-colored edges) were identified between microbial species in healthy samples. In contrast, only positive correlations were observed in stable (14) and progressing samples (21). Additionally, the total number of correlated species in healthy samples (31 species) was more than that of stable (16 species) and progressing samples (22 species). Subnetworks consisting of more than five correlated microbial species are only found in disease samples, e.g., the subnetwork consisting of five species in stable samples and that of six species in progressing periodontitis samples, respectively.

The five species of subnetwork in stable samples are Escherichia coli, Staphylococcus epidermidis, Campylobacter showae, Lactobacillus gasseri and Capnocytophaga ochracea. The seven species of subnetwork in progressing samples are Bulleidia extructa, Eubacterium infirmum, Fusobacterium periodonticum, Filifactor alocis, Gemella morbillorum, Streptococcus constellatus, 

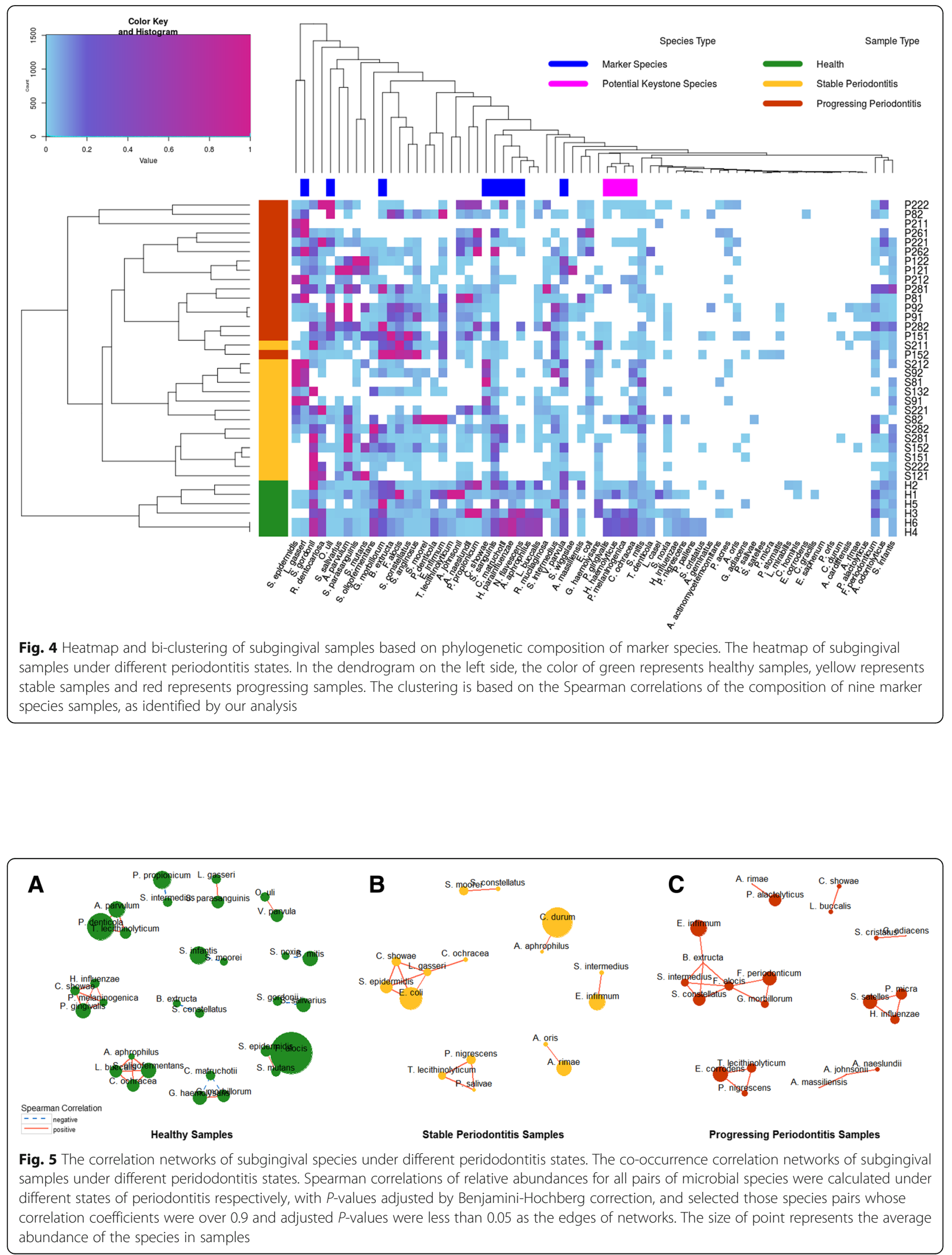
Streptococcus intermedius. Two species from the genus of Streptococcus are involved in the subnetwork of progressing samples. The overall network structure showed a potential loss of the check-and-balance mechanism through negative feedback in diseased samples.

\section{Discussion}

\section{Common core microbial species in subgingival plaques}

Overall, bacterial communities were found to be very specialized in the subgingival plaque samples. After preprocessing and profiling, an average of 47,31 and $34 \mathrm{mi}-$ crobial species were detected in healthy, stable and progressing samples, respectively. This indicates a relatively small number of species when compared to all oral microbial phyla. The numbers are consistent with those of previous reports which found as few as 50 predominant species in subgingival plaques, irrespective of health or disease $[40,41]$. These results showed that the subgingival plaques sampling procedure was carefully and conservatively performed to avoid possible contamination from the general oral environment. Based on the fact that such number has not substantially changed between their studies and ours, we conclude that the highthroughput, culture-independent methodology faithfully preserves the aboundance structure, even though it is now much more sensitive to the heterogeneity of microbiotas resident in host oral cavity.

Based on phylogenetic analysis (see Fig. 2), we identified such predominant microbial species as Streptococcus gordonii, Streptococcuss anguinis and Lactobacillus gasseri, which are consistent with those identified by Aas et al. and Paster et al. in subgingival samples [40, 41]. Since the oral cavity is the main portal through which most microorganisms enter human bodies, it is possible to detect many transient microbes in the oral environment through metagenomic techniques. Nonetheless, only a few core microbes were consistently found to inhabit subgingival plaques in both this study and those of $\mathrm{A}$ as et al. and Paster et al. [40, 41]. These results strongly suggest that periodontitis is induced by inflammatory response to bacterial challenge from the core microbes detected in subgingival biofilm [42]. Thus, the catalogue of these core microbes that persist in subgingival biofilms appears to represent the repertoire of pathogens responsible for disease onset.

In our analysis, we relied on reference genome and read mapping for composition and relative abundance estimation. It is possible that some rare species inhabiting in subgingival plaques were missed out due to low coverage of sampling procedure, low depth of read sequencing, mapping error and other random factors. However, these species' abundance should be very low even if not truly zero. In this particular study, the microbial species with a relatively high abundance are more likely to be pathogen, because periodontitis is an inflammatory disease that human immune system have active confront with microbes in subgingival plaques. Since our statistics are mainly comparing highly differentiated species, zero abundance levels due to dropouts should not have an effect. In addition, we used standardized bioinformatics pipeline to avoid bias and to estimate the microbial abundance level as accurate as possible. Almost all of the reads got mapped to the provided reference set and therefore there is not much presence of de novo species. That is because human oral microbiota has been extensively studied by clone and culture sequencing in decades, which have generated a very comprehensive set of reference sequences.

Highly abundant microbial species in subgingival plaques Among the microbial species discovered in healthy and periodontitis subgingival samples, the genus of Streptococcus was found in relative abundance. Many species of Streptococcus, such as Streptococcus gordonii, Streptococcus oligofermentans and Streptococcuss anguinis, were among the ten most prevalently abundant microbes at all status of periodontitis. This result suggests that pathogens from the genus Streptococcus may be among the most successful early colonizers to clean tooth surfaces in the human mouth by their adherence and metabolic capacities [43]. Based on their predominance in healthy samples, but decrease in periodontitis samples, their colonies might also serve as a source of biofilm adhesion for other colonizers [44].

Rothia dentocariosa was also found in high abundance in both healthy and periodontitis samples. In two of the progressing periodontitis samples, it held top abundance rank at $70.4 \%$ and $16.8 \%$, respectively, as well as in stable periodontitis samples with relative abundance of $56.2 \%$ and $32 \%$, respectively. Although Rothia species are often associated with oral health, these results are consistent with previous studies, which found that Rothia spp. can reduce oxygen levels around biofilm thus promoting the proliferation of inflammation-triggering anaerobes $[17,45]$.

Species like Atopobium parvulum, Lactobacillus gasseri, and Staphylococcus epidermidis are highly abundant in stable and progressing subgingival samples, and many of them have already been associated with periodontitis. The Atopobium genus, which is high in G + C-content and gram-positive, has previously been identified as prevalent in individuals with periodontitis, but not in healthy subjects. Lactobacillus was also found at high percentage in severe periodontitis subgingival samples [46], while Staphylococcus genus have only recently been identified as pathogens associated with periodontitis [47]. Our results further strengthen those findings.

Streptococcus mutans was also relatively abundant in our subgingival samples. This is particularly interesting 
because previous observation has shown that this microbial species can create a lactic acid-rich environment, a condition conducive to the development of symbiosis, thus making it possible for other pathogens to thrive [48].

\section{Prevalent keystone species in healthy and periodontitis subgingival plaques}

The keystone species Porphyromonas gingivalis previously identified by Orth et al. [19] was found in both healthy and diseased samples, albeit in rather small relative abundance, again hinting that this species may play a role as a keystone pathogen. A keystone pathogen typically remains functional in the background at low abundance, but promotes inflammatory state by altering host immune response to otherwise commensal microbiota. Thus, the existence of Porphyromonas gingivalis alone does not cause periodontitis. Rather, if accompanied by proper commensal microbiota, such as species from Lactobacillus, Staphylococcus and Streptococcus genera [49], in susceptible individuals, Porphyromonas gingivalis may be able to evade immune surveillance and mediate the breakdown of normal homeostatic balance in the oral environment [39].

Interestingly, Fig. 4 shows that Porphyromonas gingivalis was clustered in a small group with another three species: Haemophilus haemolyticus, Prevotella melaninogenica and Capnocytophaga ochracea. They shared similar patterns with Porphyromonas gingivalis, a widely accepted keystone pathogen, in their abundances in healthy and periodontitis samples, which indicates that these three species are likely to play a similar role similar to that of $P$. gingivalis in the oral community by significantly altering the number and community organization of oral commensal bacteria, possibly leading to periodontitis under specific conditions in susceptible bodies. However, more evidence and experimentation are needed to confirm this.

\section{Differential representation of marker species between healthy and periodontitis subgingival plaques}

Marker species are highly differentially represented in healthy and diseased subgingival plaques. As shown in Fig. 3, nine microbial species represent significant difference in relative abundance among the samples studied. In particular, Lactobacillus gasseri and Osenella uli were found to be significantly more abundant in periodontitis samples. Campylobacter showae was observed with significantly higher relative abundance in stable samples. In addition, six microbial species had significantly higher relative abundance in healthy samples compared to periodontitis samples, including Gemella morbillorum, Streptococcus sanguinis and Veillonella parvula, which were observed both in healthy and periodontitis samples, and Haemophilus parainfluenzae, Corynebacterium matruchotii and Neisseria flavescens, which were only detected in healthy samples. The $p$-values of their Dunn tests are shown in the Results section and Fig. 3.

These differentially represented species in subgingival plaques most likely compose the core microbiota directly associated with the etiology of periodontitis. As such, they could be considered markers of periodontitis. As shown in Fig. 4, when we used these nine marker microbial species as representatives of collected samples and clustered the samples based on Spearman correlations of their marker species profiles, the clustering result was nearly the same as their clinical classification (accuracy $=94.4 \%)$. This evidence strongly suggests that the combination of differentially abundant species is predictive of periodontitis at different states.

Moreover, unlike pathogen-specific diseases, the etiology of periodontitis is polymicrobial in nature and thus indicative of characteristics of the whole microbial community, or, at least, its core microbiota. It should be noted that the clustering was based on Spearman Rank-Order Correlation, rather than Euclidian distance, which gave us results consistent with clinical classification. This, in turn, means that relative hierarchy of microbial species is highly correlated with progressing periodontitis. Therefore, future studies should look for particular profile patterns of microbial composition that specifically corresponds to different states of periodontitis progression.

\section{Ecological diversity as a prognostic marker of periodontitis disease}

We then focused on the alpha-diversity of samples and its association with the progressing periodontitis. Figure 3 shows a number of microbial species with particularly high outlier abundances in both stable and progressing periodontitis subgingival samples, indicating ecological imbalance among host oral communities. When we compared alpha-diversity of samples between periodontitis and healthy samples, we found that healthy samples had the highest alpha-diversity, while stable periodontitis samples had the lowest with significant difference (see Fig. 3). Furthermore, the fitted univariate logistic model demonstrates the potential value of oral microbial alpha-diversity as a predictor of periodontitis.

\section{Polymicrobial synergy and dysbiosis (PSD) model of periodontitis etiology}

Based on recent metagenomics findings, Orth et al. proposed a Keystone-Pathogen Hypothesis which holds that keystone microbes like $P$. gingivalis may coordinate the onset and progress of periodontitis and these microbial species can alter host-microbial interactions in a manner that interrupts the homeostasis of microbial colonizers and results in inflammatory conditions that set the stage for the development of periodontitis [50]. Our results support this theory in the following way. 
According to the PSD model, periodontitis is not caused by a specific microbial species. Instead, inflammation is thought to arise from immune response to the combined activity of core and keystone pathogens. In fact, our phylogenetic profile analysis identified core pathogens and marker species showing significant abundance shifts between healthy and periodontitis samples. For example, marker species Lactobacillus gasseri, Campylobacter showae and Olsenella uli was more abundant in periodontistis samples, while the marker species Gemella morbillorum, Veillonella parvula, Haemophilus parainfluenzae, Corynebacterium matruchotii, Neisseria flavescens, Campylobacter showae and Streptococcus sanguinis were significantly more abundant in healthy samples.

We also demonstrated potential keystone species as mediators of this process (see Fig. 6). Based on the PSD model, keystone species gain virulence through cooperating with accessory microorganisms to strengthen the biofilm by creating a powerful synergistic community. Meanwhile, however, previously commensal biofilm species loses homeostasis and drop out from the competition [39, 49, 51]. Indeed, we identified the keystone species Porphyromonas gingivalis, Haemophilus haemolyticus, Prevotella melaninogenica and Capnocytophaga ochracea, all prevalent at relatively low abundance across all samples and likely acting as mediators of the observed shifts in abundance profiles between periodontitis states [49].

Thus, while these keystone species gain virulence from pathogenic associations with, for example, Lactobacillus gasseri and Osenella uli, which show relative abundance and thrive in the shifting microenvironment, other species lose ground and disappear from the oral environment, such as Haemophilus parainfluenzae, Corynebacterium matruchotii and Neisseria flavescens. Others may decrease significantly in relative abundance, such as Gemella morbillorum, Streptococcus sanguinis and Veillonella parvula. Taken together, we found Shannon diversity for all but one healthy individual to be $>2$, similar to the statistics for 10 healthy individuals reported by Bik et al. using 16S RNA sequencing data [52]. On the contrary, almost all periodontitis patients have Shannon diversity $<2$ [49]. Importantly, using alpha-diversity as an indicator of microbial flux in a state of dysbiosis, we see a decreasing pattern in periodontitis samples in accordance with the PSD model [51].

The results of our network analysis also support the PSD model and the effects of decreased diversity. The microbial species (nodes) in periodontitis samples have much less interconnectivity (edges), in particular negative associations (blue dashed edges), representing a loss of check-and-balance. There are subnetworks consisting of $>5$ correlational microbial species found only in periodontitis samples indicating the pathobionts and keystones thriving through synergistic interaction in the inflammatory state, as suggested in the preceding section. The component species in these subnetworks are different, indicating that the keystones and pathogens active in different states of periodontitis may be different. At this point, the alpha-diversity of subgingival samples has also reached the lowest level.

\section{Implications of reduced ecological diversity in periodontitis}

Irrespective of specific etiology, this and other studies have found oral microbial alpha-diversity to be strongly negatively correlated with periodontitis status [50].

Mutualism and commensalism are hallmarks of diversity among the aggregate of microorganisms resident in human, and such diversity is generally indicative of health [53]. This principle has been tested under conditions of dysbiosis in mucosal diseases of the gastrointestinal tract, such as inflammatory bowel disease and

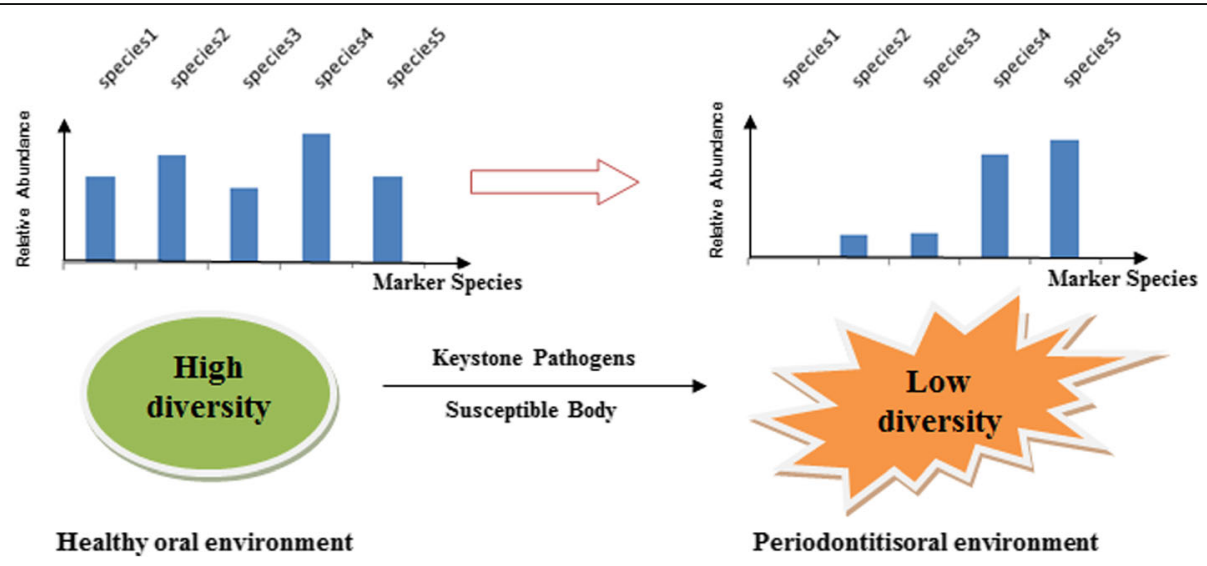

Fig. 6 The keystone pathogens mediated polymicrobial synergy and dysbiosis model of periodontitis 
obesity, both considered to be associated with decreased diversity of microbial community [54]. Some researchers, however, have reported that the diversity of oral microbiota is higher in periodontitis compared to healthy subjects $[55,56]$.

We offered evidence in the present metagenomics study indicating that the principle microbiome diversity is also applicable to periodontal health. Indeed, a healthy oral microbiome is generally reflective of bacterial homeostasis, featuring both synergistic and antagonistic interactions between microbes and between microbes and host [14]. From our results, however, it is likely that polymicrobial synergy eventually leads to dysbiosis by the breakdown of ecological balance in periodontal microbial communities and that the disease state follows.

Accordingly, our results could potentially offer a novel diagnostic and prognostic paradigm based on establishing classification models to distinguish among different states of periodontitis using alpha-diversity and species composition as metrics, together with a sufficient database. However, since the results of the present study cannot pinpoint community diversity as either a cause or result of periodontitis, further study is required to determine whether alpha-diversity of subgingival plaques can, indeed, predict incipient periodontitis in individuals whose clinical symptoms have not yet manifested.

It should be noted that our study was limited in sample size and that we only used average abundances in network construction to reflect the general scope of the oral community in subgingival plaques under different states of periodontitis. However, if longitudinal metagenomics data were to be collected for each periodontitis subject in a larger cohort study, individual oral networks could be constructed, and the underlying correlations among those networks under particular states of periodontitis could be detected. This would provide a better understanding of the etiology of periodontitis.

\section{Conclusion}

This study aimed to discover potential patterns and interactions among microbial communities under three states: healthy control, stable periodontitis, and progressing periodontitis. We integrated metagenomic sequence data of subgingival plaque samples collected from 43 subjects in two previous studies, including Duran-Pinedo et al. [20] and Yost et al. [21]. We found alpha-diversity to be the single strongest predictor of subjects' periodontitis status $(P<0.011)$ such that healthy subjects had the highest alpha-diversity, while subjects with stable sites had the lowest. We also found that the phylogenetic profiles of nine marker microbes could be applied to differentiate the states of periodontitis to an accuracy of $94.4 \%$. We showed the reduction of negatively correlated species to be another notable signature of disease progression.
Overall, our results support the polymicrobial synergy and dysbiosis model of periodontitis etiology by exploring pathogenic factors that impact microbial homeostasis versus homeostatic imbalance in oral microbiota communities. More importantly, our results consistently show a strong association between the loss of oral microbiota diversity and disease status based on evidence from phylogenetic diversity, clustering and network analyses. This reduction of alpha-diversity is an event distinct from actual etiology. This suggests that metagenomics sequencing can produce phylogenetic profiling results predictive of periodontal health status, possibly leading to novel therapeutic modalities.

\section{Acknowledgements}

We thank David Martin for editorial assistance in English language.

\section{Declarations}

This article has been published as part of BMC Genomics Volume 18 Supplement 1, 2016: Proceedings of the 27th International Conference on Genome Informatics: genomics. The full contents of the supplement are available online at http://bmcgenomics.biomedcentral.com/articles/ supplements/volume-18-supplement-1.

\section{Funding}

The authors are supported by the National Natural Science Foundation of China (61370131). LCX is additionally supported by U.S. National Institutes of Health of (2R01HG006137-04). The publication costs were funded by the National Natural Science Foundation of China (61370131).

\section{Availability of data and materials}

The two metagenomic datasets are all publicly available in the supplementary of their original publications [20, 21]. The GRAMMy [27] software package that implements the refined phylogenetic analysis is described in and freely available for academic use from the website: http:// bitbucket.org/charade/grammy. All other software or programs used in this analysis are also freely available (please find web links in their publications).

\section{Authors' contributions}

$\mathrm{DA}, \mathrm{RH}, \mathrm{LCX}$ conceived and designed the study. DA, RH analyzed the data. All authors contributed to and approved the final manuscript.

\section{Competing interests}

The authors declare that they have no competing interests.

\section{Consent for publication}

Not applicable.

\section{Ethics approval and consent to participate}

The two metagenomics datasets we used were publicly available and their ethics approval and informed consents were all found in their original publications [20, 21], which allow for academic research use. No additional ethics approval and informed consent were required for the study, because this study did not involve the use of any new human and animal subjects, human tissue and human data.

\section{Author details}

${ }^{1}$ School of Mathematics and Physics, University of Science and Technology Beijing, 30 Xueyuan Road, Haidian District, Beijing 100083, People's Republic of China. '2Department of Prosthodontics, Ninth People's Hospital Affiliated with Shanghai Jiao Tong University, School of Medicine, 639 Zhizaoju Road, Shanghai 200011, China. ${ }^{3}$ Oral Bioengineering Lab, Shanghai Research Institute of Stomatology, Ninth People's Hospital Affiliated with Shanghai Jiao Tong University, School of Medicine, Shanghai Key Laboratory of Stomatology, 639 Zhizaoju Road, Shanghai 200011, China. ${ }^{4}$ Department of Medicine, Stanford University School of Medicine, 269 Campus Dr., Stanford, 
CA 94305, USA. ${ }^{5}$ Department of Statistics, The Wharton School, University of Pennsylvania, 3730 Walnut Street, Philadelphia, PA 19014, USA.

\section{Published: 25 January 2017}

\section{References}

1. Kajiya M, Giro G, Taubman MA, Han X, Mayer MP, Kawai T. Role of periodontal pathogenic bacteria in RANKL-mediated bone destruction in periodontal disease. J Oral Microbiol. 2010;2:5532.

2. Ali J, Pramod K, Tahir MA, Ansari SH. Autoimmune responses in periodontal diseases. Autoimmun Rev. 2011;10:426-31.

3. Pérez-Losada FL1, Jané-Salas E, Sabater-Recolons MM, Estrugo-Devesa A, Segura-Egea JJ, López-López J. Correlation between periodontal disease management and metabolic control of type 2 diabetes mellitus. A systematic literature review. Med Oral Patol Oral Cir Bucal. 2016; 31:0.

4. Teles R, Wang CY. Mechanisms involved in the association between peridontal diseases and cardiovascular disease. Oral Dis. 2011;17:450-61.

5. Eke PI, Dye BA, Wei L, Thornton-Evans GO, Genco RJ. Prevalence of periodontitis in adults in the United States: 2009 and 2010. J Dent Res. 2012:91:914-20

6. Jenkinson HF. Beyond the oral microbiome. Environ Microbio. 2011;13:3077-87.

7. Paster BJ, Olsen I, Aas JA, Dewhirst FE. The breadth of bacterial diversity in the human periodontal pocket and other oral sites. Periodontol 2000. 2006;42:80-7.

8. Loesche WJ, Nafe D. Reduction of supragingival plaque accumulations in institutionalized Down's syndrome patients by periodic treatment with topical kanamycin. Arch Oral Biol. 1973;18:1131-43.

9. Holt SC, Ebersole JL. Porphyromonas gingivalis, Treponema denticola, and Tannerella forsythia: the 'red complex', a prototype polybacterial pathogenic consortium in periodontitis. Periodontology. 2005;38:72-122.

10. Socransky SS, Haffajee AD, Cugini MA, Smith C, Kent Jr RL. Microbial complexes in subgingival plaque. J Clin Periodontol. 1998;25:134-44.

11. Kumar PS, Griffen AL, Barton JA, Paster BJ, Moeschberger ML, Leys EJ. New bacterial species associated with chronic periodontitis. J Dent Res. 2003;82:338-44.

12. Theilade $E$. The non-specific theory in microbial etiology of inflammatory periodontal diseases. J Clil Periodontol. 1986;13:905-11.

13. Marsh PD. Microbial ecology of dental plaque and its significance in health and disease. Adv Dent Res. 1994;8:263-71

14. Marsh PD. Are dental diseases examples of ecological catastrophes? Microbiology. 2003;149:279-94.

15. Turnbaugh PJ, Ley RE, Mahowald MA, Magrini V, Mardis ER, Gordon Jl. An obesity-associated gut microbiome with increased capacity for energy harvest. Nature. 2006:444:1027-31.

16. Qin J, Li R, Raes J, Arumugam M, Burgdorf KS, Manichanh C, Nielsen T, et al. A human gut microbial gene catalogue established by metagenomic sequencing. Nature. 2010;464:59-65.

17. Abusleme L, Dupuy AK, Dutzan N, Silva N, Burleson JA, Strausbaugh LD, Gamonal J, Diaz PI. The subgingival microbiome in health and periodontitis and its relationship with community biomass and inflammation. ISME J. 2013;7:1016-25

18. Wang J, Qi J, Zhao H, He S, Zhang Y, Wei S, Zhao F. Metagenomic sequencing reveals microbiota and its functional potential associated with periodontal disease. Sci Rep. 2013;3:1843.

19. Orth RK, O'Brien-Simpson N, Dashper SG, Reynolds EC. Synergistic virulence of Porphyromonas gingivalis and Treponema denticola in a murine periodontitis model. Mol Oral Microbiol. 2011;26:229-40.

20. Duran-Pinedo AE, Chen T, Teles R, Starr JR, Wang X, Krishnan K, et al. Community-wide transcriptome of the oral microbiome in subjects with and without periodontitis. ISME J. 2014;8:1659-72.

21. Yost S, Duran-Pinedo AE, Teles R, Krishnan K, Frias-Lopez J. Functional signatures of oral dysbiosis during periodontitis progression revealed by microbial metatranscriptome analysis. Genome Med. 2015;7:27.

22. Schmieder R, Lim YW, Rohwer F, Edwards R. TagCleaner: identification and removal of tag sequences from genomic and metagenomic datasets. BMC Bioinformatics. 2010;11:341.

23. Schmieder R, Edwards R. Quality control and preprocessing of metagenomic datasets. Bioinformatics. 2011;27:863-4.

24. Schmieder R, Edwards R. Fast identification and removal of sequence contamination from genomic and metagenomic datasets. PLoS One. 2011;6, e17288.
25. Magoč T, Salzberg SL. FLASH: fast length adjustment of short reads to improve genome assemblies. Bioinformatics. 2011;27:2957-63.

26. Segata N, Waldron L, Ballarini A, Narasimhan V, Jousson O, Huttenhower C. Metagenomic microbial community profiling using unique clade-specific marker genes. Nat Methods. 2012;9:811-4.

27. Xia LC, Cram JA, Chen T, Fuhrman JA, Sun F. Accurate genome relative abundance estimation based on shotgun metagenomic reads. PLoS One. 2011;6, e27992.

28. Markowitz VM, Ivanova NN, Szeto E, Palaniappan K, Chu K, Dalevi D, et al. IMG/M: a data management and analysis system for metagenomes. Nucleic Acids Res. 2008:36:D534-8.

29. Chen T, Yu WH, Izard J, Baranova OV, Lakshmanan A, Dewhirst FE. The Human Oral Microbiome Database: a web accessible resource for investigating oral microbe taxonomic and genomic information. Database. 2010;2010:baq013.

30. Li H, Durbin R. Fast and accurate short read alignment with BurrowsWheeler transform. Bioinformatics. 2009;25:1754-60.

31. Benjamini $Y$, Hochberg Y. Controlling the false discovery rate: a practical and powerful approach to multiple testing. J R Stat Soc Series B Stat Methodol. 1995;57(1):289-300.

32. Legendre P, Gallagher ED. Ecologically meaningful transformations for ordination of species data. Oecologia. 2001;129(2):271-80.

33. Yao Y, Charlesworth J, Nair V, Watson M. MicroRNA expression profiles in avian haemopoietic cells. Front Genet. 2013;4:153.

34. Xia LC, Steele JA, Cram JA, Cardon ZG, Simmons SL, Vallino JJ, et al. Extended local similarity analysis (eLSA) of microbial community and other time series data with replicates. BMC Syst Biol. 2011;5:1.

35. Weiss S, Van Treuren W, Lozupone C, Faust K, Friedman J, Deng Y, et al. Correlation detection strategies in microbial data sets vary widely in sensitivity and precision. ISME J. 2016;10:1669-81.

36. Steele JA, Countway PD, Xia L, Vigil PD, Beman JM, Kim DY, et al. Marine bacterial, archaeal and protistan association networks reveal ecological linkages. ISME J. 2011;5:1414-25.

37. Xia LC, Ai D, Cram J, Fuhrman JA, Sun F. Efficient statistical significance approximation for local similarity analysis of high-throughput time series data. Bioinformatics. 2013;29:230-7.

38. Xia LC, Ai D, Cram JA, Liang X, Fuhrman JA, Sun F. Statistical significance approximation in local trend analysis of high-throughput time-series data using the theory of Markov chains. BMC Bioinformatics. 2015;16:1.

39. Hajishengallis G, Darveau RP, Curtis MA. The keystone-pathogen hypothesis. Nat Rev Microbiol. 2012;10:717-25.

40. Aas JA, Paster BJ, Stokes LN, Olsen I, Dewhirst FE. Defining the normal bacterial flora of the oral cavity. J Clin Microbio. 2005;43:5721-32.

41. Paster BJ, Boches SK, Galvin JL, Ericson RE, Lau CN, Levanos VA, et al. Bacterial diversity in human subgingival plaque. J Bacterio. 2001;183:3770-83.

42. Curtis MA, Zenobia C, Darveau RP. The relationship of the oral microbiotia to periodontal health and disease. Cell Host Microbe. 2011;10(4):302-6.

43. Nobbs AH, Lamont RJ, Jenkinson HF. Streptococcus adherence and colonization. Microbiol Mol Biol Rev. 2009;73:407-50.

44. Kolenbrander PE, Palmer RJ, Periasamy S, Jakubovics NS. Oral multispecies biofilm development and the key role of cell-cell distance. Nat Rev Microbiol. 2010:8:471-80.

45. Kistler JO, Booth V, Bradshaw DJ, Wade WG. Bacterial community development in experimental gingivitis. PLoS One. 2013;8, e71227.

46. Moore WE, Holdeman LV, Smibert RM, Hash DE, Burmeister JA, Ranney RR. Bacteriology of severe periodontitis in young adult humans. Infectlmmun. 1982;38:1137-48.

47. da Silva-Boghossian CM, do Souto RM, Luiz RR, Colombo AP. Association of red complex, A. actinomycetemcomitans and non-oral bacteria with periodontal diseases. Arch Oral Biol. 2011:56:899-906.

48. Kanasi E, Dewhirst FE, Chalmers NI, Kent Jr R, Moore A, Hughes CV, et al. Clonal analysis of the microbiota of severe early childhood caries. Caries Res. 2010:44:485-97.

49. Hajishengallis G, Liang S, Payne MA, Hashim A, Jotwani R, Eskan MA, et al. Low-abundance biofilm species orchestrates inflammatory periodontal disease through the commensal microbiota and complement. Cell Host Microbe. 2011:10:497-506.

50. Hajishengallis G. Immunomicrobial pathogenesis of periodontitis: keystones, pathobionts, and host response. Trends Immunol. 2014;35:3-11. 
51. Hajishengallis G, Lamont RJ. Beyond the red complex and into more complexity: the polymicrobial synergy and dysbiosis (PSD) model of periodontal disease etiology. Mol Oral Microbiol. 2012;27:409-19.

52. Bik EM, Long CD, Armitage GC, Loomer P, Emerson J, Mongodin EF, et al. Bacterial diversity in the oral cavity of 10 healthy individuals. ISME J. 2010;: :962-74

53. Rosier BT, De Jager M, Zaura E, Krom BP. Historical and contemporary hypotheses on the development of oral diseases: are we there yet. Front Cell Infect Microbiol. 2014;4:92.

54. Walker AW, Sanderson JD, Churcher C, Parkes GC, Hudspith BN, Rayment N, Brostoff J, Parkhill J, Dougan G, Petrovska L. High-throughput clone library analysis of the mucosa-associated microbiota reveals dysbiosis and differences between inflamed and non-inflamed regions of the intestine in inflammatory bowel disease. BMC Microbiol. 2011;11(1):7.

55. Griffen AL, Beall CJ, Campbell JH, Firestone ND, Kumar PS, Yang ZK, et al. Distinct and complex bacterial profiles in human periodontitis and health revealed by 165 pyrosequencing. ISME J. 2012;6:1176-85.

56. Liu B, Faller LL, Klitgord N, Mazumdar V, Ghodsi M, Sommer DD, et al. Deep sequencing of the oral microbiome reveals signatures of periodontal disease. PLoS One. 2012;7, e37919.

Submit your next manuscript to BioMed Central and we will help you at every step:

- We accept pre-submission inquiries

- Our selector tool helps you to find the most relevant journal

- We provide round the clock customer support

- Convenient online submission

- Thorough peer review

- Inclusion in PubMed and all major indexing services

- Maximum visibility for your research

Submit your manuscript at www.biomedcentral.com/submit
Biomed Central 